\title{
\begin{tabular}{l|l} 
Mitraries & DSpace@MIT
\end{tabular}
}

\author{
MIT Open Access Articles
}

\section{Attosecond Resolution Timing Jitter Characterization of Diode Pumped Femtosecond Cr:Lisaf Lasers}

The MIT Faculty has made this article openly available. Please share how this access benefits you. Your story matters.

Citation: Umit Demirbas et al. "Attosecond resolution timing jitter characterization of diode pumped femtosecond Cr:LiSAF lasers" Conference on Lasers and Electro-Optics (CLEO) and Quantum Electronics and Laser Science Conference (QELS), 2010. (C) Copyright 2010 IEEE

As Published: http://ieeexplore.ieee.org/xpl/articleDetails.jsp? $\mathrm{tp}=$ \&arnumber $=5500819 \&$ contentType=Conference+Publications\&searchField\%3DSearch_All

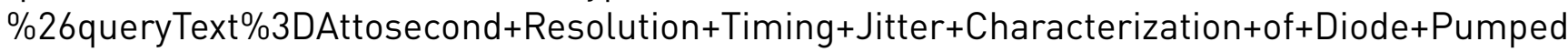
+ Femtosecond+Cr\%3ALisaf+Lasers

Publisher: Institute of Electrical and Electronics Engineers (IEEE)

Persistent URL: http://hdl.handle.net/1721.1/74003

Version: Final published version: final published article, as it appeared in a journal, conference proceedings, or other formally published context

Terms of Use: Article is made available in accordance with the publisher's policy and may be subject to US copyright law. Please refer to the publisher's site for terms of use. 


\title{
Attosecond resolution timing jitter characterization of diode pumped femtosecond Cr:LiSAF lasers
}

\author{
Umit Demirbas, Andrew Benedick, Alphan Sennaroglu, Duo Li, \\ Jungwon Kim, James G. Fujimoto, and Franz X. Kärtner \\ ${ }^{1}$ Department of Electrical Engineering and Computer Science and Research Laboratory of Electronics, \\ Massachusetts Institute of Technology, 77 Massachusetts Avenue, Cambridge, MA 02139 \\ igfuji@mit.edu and kaertner@mit.edu
}

\begin{abstract}
Two diode pumped Cr:LiSAF lasers are synchronized using a balanced nonlinear optical cross correlator. An integrated timing jitter of less than 156 as in the $10 \mathrm{kHz}$ to $10 \mathrm{MHz}$ range is measured. 2010 Optical Society of America

OCIS codes: (320.7090) Ultrafast lasers; (320.7160) Ultrafast technology
\end{abstract}

Accurate measurement of timing jitter noise of optical pulse trains from mode-locked lasers is a challenging task, due to their inherent ultralow noise characteristics. For typical solid-sate mode-locked lasers, theoretical analysis estimates a timing jitter in the attosecond regime at high frequencies $(10 \mathrm{kHz})[1,2]$; whereas, it is quite challenging to achieve sub-10-fs resolution in a timing jitter measurement. The most common measurement method uses a high-speed photodetector to generate a microwave signal, which is then bandpass filtered at higher harmonics, and a microwave mixer measures the phase noise of the selected harmonic [3]. Although this method is often employed, excess phase noise may be generated in the photodetection process and the measurement accuracy is inherently limited by the resolution of the microwave mixer [4]. Another method for measuring timing jitter noise is interferometric cross correlation [5], which allows higher sensitivity measurements. However, this method has a limited measurement range (less than an optical cycle), making it hard to use for free running mode-locked lasers. Moreover, this method measures optical phase noise rather than the timing jitter noise. Balanced nonlinear optical intensity cross correlation is an all optical domain technique which can precisely measure the timing jitter noise of mode-locked lasers in a simple and direct way $[4,6]$. Balanced nonlinear cross correlation has high sensitivity, sufficient detection range, and does not suffer from excess photodetection noise. Furthermore, balanced photodetection of the cross-correlation signal greatly reduces coupling of laser intensity noise to the measured timing jitter.

In this work, we present attosecond resolution timing jitter characterization of femtosecond diode pumped Cr:LiSAF lasers by using a single crystal balanced optical intensity cross correlator. Cr:LiSAF lasers are low-cost, compact, efficient, enable turn-key operation and are expected to show ultra-low timing jitter [7]. Two home-built, diode-pumped, saturable absorber mirror mode-locked, $100 \mathrm{MHz}$ repetition rate Cr:LiSAF lasers, were synchronized using a nonlinear optical cross correlator in a low-bandwidth ( $1 \mathrm{kHz}$ ) phase-locked loop (PLL) configuration. The resulting timing jitter noise spectral density between the two lasers was observed. Since the two lasers where identically constructed, the timing jitter measured outside the locking bandwidth reflects $\sqrt{2}$ times the timing jitter of an individual laser. The balanced nonlinear optical cross correlator provides a measurement resolution of $2 \times 10^{-10} \mathrm{fs}^{2} / \mathrm{Hz}$ at $100 \mathrm{kHz}$ $(-195 \mathrm{dBc}$ at $1 \mathrm{GHz}$ carrier). The upper limit for the integrated timing jitter of the Cr:LiSAF lasers was measured to be 156 as in [10 kHz-10 MHz]. To the best of our knowledge, this is the lowest reported value of timing jitter for any mode-locked solid-state laser, and demonstrates the inherently low noise character of mode-locked lasers at high frequencies. This study shows the potential of low-cost fs Cr:LiSAF laser systems as ultra-low timing jitter sources [7], and the advantage of the balanced optical cross correlation for timing jitter measurements $[4,6]$.

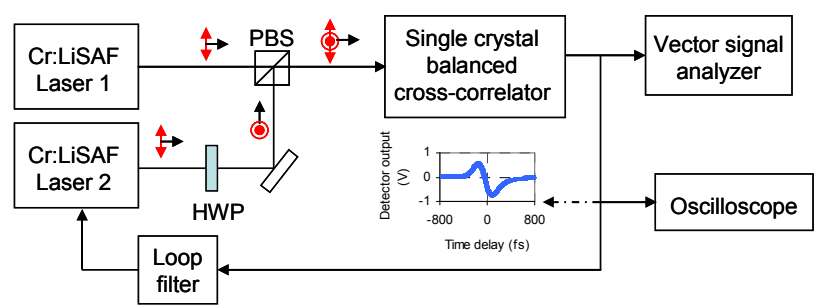

(a)

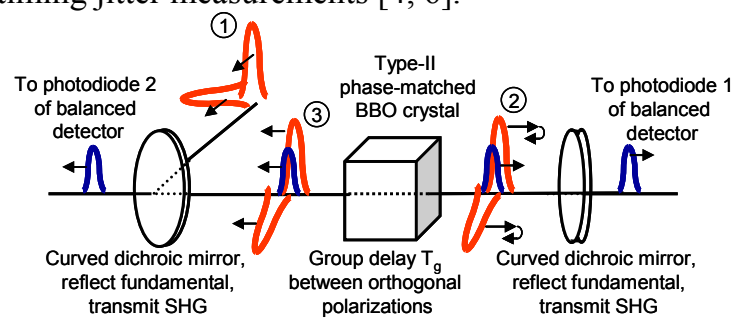

(b)

Fig. 1. (a) Schematic of the timing detector method. (b) Implementation of single crystal balanced optical cross correlator.

Fig. 1 shows the schematic of the timing jitter noise measurement using balanced optical cross correlation. Two home-built, diode pumped, saturable absorber mode-locked, $100 \mathrm{MHz} \mathrm{Cr}$ :LiSAF lasers provided about 100-fs long 1$\mathrm{nJ}$ pulses around $850 \mathrm{~nm}$. The output beams are combined using a half-wave plate (HWP) and a polarizing beam splitter cube (PBS). The combined laser output is sent to the first dichroic mirror of the cross correlator, which focuses the beams inside a $400 \mathrm{~m}$ thick type-II phase matching optimized BBO crystal for $850 \mathrm{~nm}$. The second harmonic (SH) generated in the forward pass is transmitted through the second dichroic mirror and then directed to the first photodiode 


\section{CTuDD6.pdf}

of the balanced detector (New Focus 2107-FS). The nonlinear crystal also provides group delay ( $\mathrm{T}_{\mathrm{g}} 100 \mathrm{fs}$ ) between the orthogonal polarizations. The returning beams (at the fundamental) are focused back in to the nonlinear crystal, where the SH is generated and detected by the second photodiode of the balanced detector. The signal from the balanced detector is proportional to the timing delay between the two pulses. The cross correlator signal at low frequencies is also used as the error signal for the PLL, which synchronizes the lasers by actuating a small PZT in one of the lasers. Once the lasers are synchronized with the low-bandwidth PLL, the signal in the high frequency range is directly proportional to the relative timing jitter of the free running lasers, which is uncorrelated $[4,6]$.

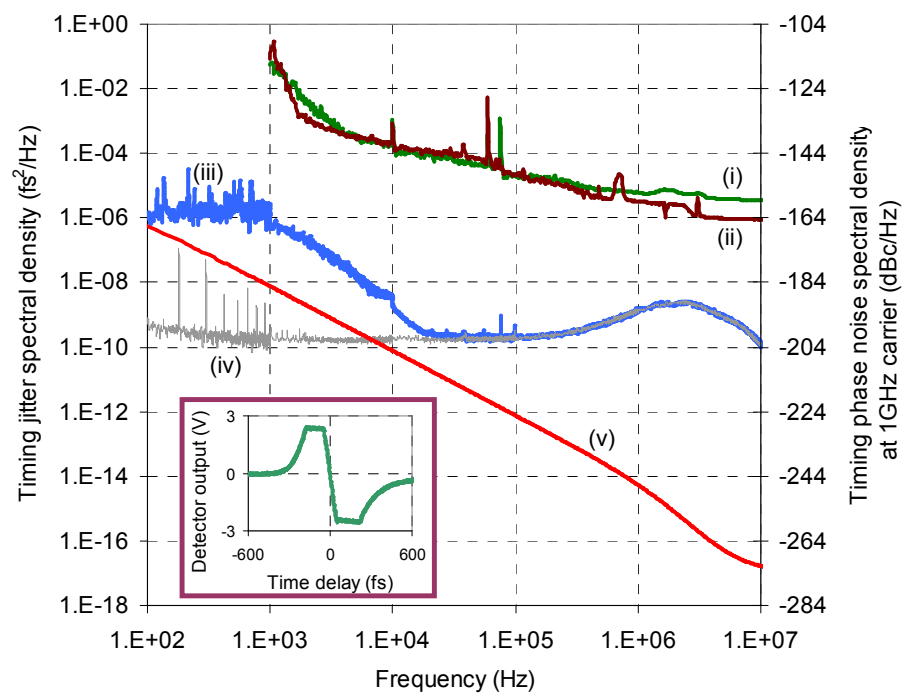

Fig. 2. Timing jitter spectral density measurement between two Cr:LiSAF lasers. Secondary axis shows the corresponding phase noise referenced to a $1 \mathrm{GHz}$ carrier. (i)-(ii): Measured jitter density of laser 1 and 2 using the signal source analyzer. (iii): Measured jitter density between laser 1 and 2 using the balanced cross correlator setup. (iv): Background noise level of the balanced cross correlator measurement. (v): Theoretically estimated timing jitter spectral density (for one laser). Inset: Measured cross correlation curve when the lasers are not synchronized.

Fig. 2 summarizes the timing jitter measurement results. Curves (i) and (ii) show the jitter density measurement of laser 1 and 2 using a commercial signal source analyzer (Agilent E5052B) and using the $10^{\text {th }}$ harmonic at $1 \mathrm{GHz}$. The result indicates an integrated timing jitter of $10 \mathrm{fs}$ from $10 \mathrm{kHz}$ to $10 \mathrm{MHz}$, which is limited by the signal source analyzer. The measured noise level is orders of magnitude higher then the theoretically estimated noise level (curve (v)). Curve (iii) shows the measured timing jitter spectral density between both lasers using the balanced nonlinear optical cross correlator. Curve (iv) shows the background level for this measurement, which is dominated by the resonance around $2.5 \mathrm{MHz}$ that is due to the non-ideal frequency response of the balanced detector. The estimated theoretical jitter density level due to quantum noise for the Cr:LiSAF lasers is also plotted in Fig. 2 (curve (v)), where the integrated timing jitter from $10 \mathrm{kHz}$ to $10 \mathrm{MHz}$ is about 1.3 as. The measured integrated timing jitter from $10 \mathrm{kHz}$ to 10 $\mathrm{MHz}$ is 156 as. From the background measurement (iv), we can conclude, that only a few tens of attoseconds maybe due to the actual timing jitter of the laser in the range from 1-10 kHz. The rest of the measurement is due to the detector background. This measurement clearly demonstrates, that the quantum limited timing jitter of short pulse solid-state lasers beyond $10 \mathrm{kHz}$ is in the sub-100 as and potentially even below 10 as. Further studies will focus on improving the sensitivity by optimizing the SH generation efficiency and by using an improved balanced detector to truly detect quantum limited performance.

\section{References}

[1] S. Namiki and H. A. Haus, "Noise of the stretched pulse fiber laser .1. Theory," IEEE Journal of Quantum Electronics, vol. 33, pp. 649659, May 1997.

[2] R. Paschotta, "Noise of mode-locked lasers (Part II): timing jitter and other fluctuations," Applied Physics B-Lasers and Optics, vol. 79, pp. 163-173, Jul 2004.

[3] R. P. Scott, C. Langrock, and B. H. Kolner, "High-dynamic-range laser amplitude and phase noise measurement techniques," IEEE Journal of Selected Topics in Quantum Electronics, vol. 7, pp. 641-655, Jul-Aug 2001.

[4] J. Kim, J. Chen, J. Cox, and F. X. Kärtner, "Attosecond-resolution timing jitter characterization of free-running mode-locked lasers," Optics Letters, vol. 32, pp. 3519-3521, Dec 152007.

[5] Y. F. Chen, J. Jiang, and D. J. Jones, "Remote distribution of a mode-locked pulse train with sub 40-as jitter," Optics Express, vol. 14, pp. 12134-12144, Dec 112006.

[6] T. R. Schibli, J. Kim, O. Kuzucu, J. T. Gopinath, S. N. Tandon, G. S. Petrich, L. A. Kolodziejski, J. G. Fujimoto, E. P. Ippen, and F. X. Kärtner, "Attosecond active synchronization of passively mode-locked lasers using balanced cross-correlation," Opt. Lett., vol. 28, pp. 947-949, 2003.

[7] U. Demirbas, D. Li, J. R. Birge, A. Sennaroglu, G. S. Petrich, L. A. Kolodziejski, F. X. Kärtner, and J. G. Fujimoto, "Low-cost, singlemode diode-pumped Cr:Colquiriite lasers " Optics Express, vol. 17, pp. 14374-14388, 2009. 